\title{
Spontaneous rupture of uterine surface vessel from a multigravida in spontaneous labor
}

\author{
Juliana Quay, Azra Naseem, George Kaladelfos
}

\section{ABSTRACT}

Introduction: Spontaneous rupture of a uterine vessel during labor is rare Case Report:A 41-yearold multigravida presented with spontaneous hemoperitoneum one day after spontaneous vaginal delivery with no prior history of cesarean section. Laparotomy revealed a bleeding vessel on the right lateral posterior aspect of the uterine serosa, which led to hemoperitoneum causing hemorrhagic shock and a spontaneous surgical abdomen. Conclusion: uterine surface vessel rupture is rare and difficult to diagnose. However with close observation to vital signs and symptoms, immediate resuscitative efforts could be taken.

Keywords: Hemoperitoneum, Labor, Pregnancy, Spontaneous uterine vessel rupture

\section{How to cite this article}

Quay J, Naseem A, Kaladelfos G. Spontaneous rupture of uterine surface vessel from a multigravida in spontaneous labor. Case Rep Int 2015;4:43-46.

Juliana Quay ${ }^{1}$, Azra Naseem ${ }^{2}$, George Kaladelfos ${ }^{3}$ Affiliations: ${ }^{1} \mathrm{MB} \mathrm{ChB}$, Registrar, Department of Obstetrics and Gynecology, Maitland Hospital, Maitland, NSW, Australia; ${ }^{2 M B B S}$, FCPS, MRCOG, FRANZCOG, Staff Specialist Consultant, Department of Obstetrics and Gynecology, Maitland Hospital, Maitland, NSW, Australia; ${ }^{3} \mathrm{MBBS}$, BSc (Hons), FRANZCOG, Specialist Consultant, Department of Obstetrics and Gynecology, Maitland Hospital, Maitland, NSW, Australia.

Corresponding Author: Juliana Quay, 4/21 Ranclaud Street Merewether, Newcastle, New South Wales 2291, Australia. Ph: (0061)478155662; Email: juliana.quay@gmail.com

Received: 06 April 2015

Accepted: 23 May 2015

Published: 07 October 2015
Article ID: 100016CRINTJQ2015

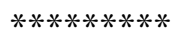

doi:10.5348/crint-2015-16-CR-11

\section{INTRODUCTION}

Spontaneous rupture of a uterine vessel during labor is rare. There are less than 150 reportable cases of spontaneous uterine vessel rupture in English literature [1-8]. There have been documented cases of uterine vessel rupture in twin pregnancy and in patients with endometriosis. Here we report the first case of its kind in a grand multigravida who had a normal vaginal delivery then became peritonitic with hypovolemic shock one day postpartum. There was no evidence of endometriosis in this case. During operation it was noted that she had a spontaneous surface vessel rupture from the right lateral and posterior aspect of her uterus. This caused internal bleeding which led to hemoperitoneum and hemodynamic instability.

\section{CASE REPORT}

A 41-year-old female gravida 6 para 5 at $38+5$ weeks gestation was transferred down from a rural referral hospital in early stages of labor.

The patient had five previous normal vaginal deliveries at term. Her BMI was 39. She had gestational diabetes (GDM) managed on insulin. Her morphology scan was late at 28 weeks gestation. It was normal with a fundal placenta. She was otherwise antenatally well. Her past medical/surgical history includes cholecystectomy and a right salpingo-oophorectomy (RSO) for a large ovarian cyst five years ago for a mucinous cystadenoma.

The patient was seen in the birthing unit the following morning and a vaginal examination was performed. Whilst it was a difficult exam due to patient discomfort, she was assessed to be $7 \mathrm{~cm}$ dilated with bulging membranes and 
the presenting part at station-3. She had continuous CTG monitoring because of an atypical deceleration at the beginning of labor.

Four hours later, she was assessed for cervical progress during labor. She had to inhale nitrous oxide gas for analgesia during the vaginal examination as she had intense discomfort from the assessment earlier. During her vaginal examination, she cried out in severe distress and had a brief period of loss of consciousness (LOC). A Medical Emergency Response Team (MET) was called. Her vitals were stable apart from an elevated pulse rate. It was measured between $110-120 \mathrm{bpm}$. The rest of her clinical examination was normal. Her LOC and tachycardia was attributed to stress reaction from the discomfort of the vaginal examination and from labor.

Vaginal examination of the patient was repeated once she was stable and an artificial rupture of membranes (ARM) was performed. Her amniotic liquor was clear on ARM. She continued to labor for the next four hours with very close supervision and continuous monitoring of vitals and CTG. Her CTG was suspicious with atypical variable decelerations though baseline rate of the trace was maintained and its variability greater than 5 (Figure 2). After $4^{1 / 2}$ hours of ARM, she commenced pushing and delivered a live baby boy 6 minutes after active second stage. The baby came out floppy with no respiratory effort but had a HR>100. It had CPAP for 30 seconds and IPPV for 1 minute. Its APGARs were 3 at 1 st minute, 9 at 5 th minute and 10 at 10 th minute. The placenta was delivered via controlled cord traction and the patient had an intact perineum with minimal per vaginal (PV) loss and a firm and contracted uterus. Of note, she remained tachycardic with a pulse rate (PR) 113-123 bpm but normotensive blood pressure 148/90 mmHg.

The patient was reviewed soon after delivery, Her blood pressure was 85/46 and tachycardia PR was 135 . Her abdomen was soft and not tender. Her fundus was firm and contracted below her umbilicus and she had minimal PV loss. Her perineum was reviewed again and there were no tears and no clots expressed with fundal pressure. An indwelling catheter (IDC) was inserted which drained concentrated urine. Her vitals responded to bolus intravenous therapy (IVT) and her blood pressure became 120/75 and her PR 117. An hour later, she had a further review for tachycardia PR 130. She was normotensive and was otherwise well and comfortable. A full blood count was taken then and her hemoglobin was $7.2 \mathrm{~g} / \mathrm{L}$. This was a marked fall from a level of hemoglobin $11.8 \mathrm{~g} / \mathrm{L}$ two days prior when she was transferred from the rural hospital. She was transfused with 3 units of packed red cell and a general medical review was requested for the unexplained persistent tachycardia with no obvious evidence of postpartum hemorrhage and an urgent ultrasound scan was organized. By this time she had some abdominal tenderness.

Ultrasound revealed large hemoperitoneum. The suspicion was a ruptured uterus. She was immediately taken to the operating theatre for a laparotomy (Figure 1).
During the operation, the patient had a large amount of blood in the abdomen, which was aspirated. Her uterus was then found to be completely intact. The source of bleeding was from a bleeder in the right lateral and posterior aspect of the uterine serosa in the region of the lower segment which had hemorrhaged into the peritoneum and into right broad ligament. The bleeder was ligated with figure of 8 sutures. She needed right uterine artery ligation to achieve hemostasis. A massive transfusion protocol was initiated at laparotomy.
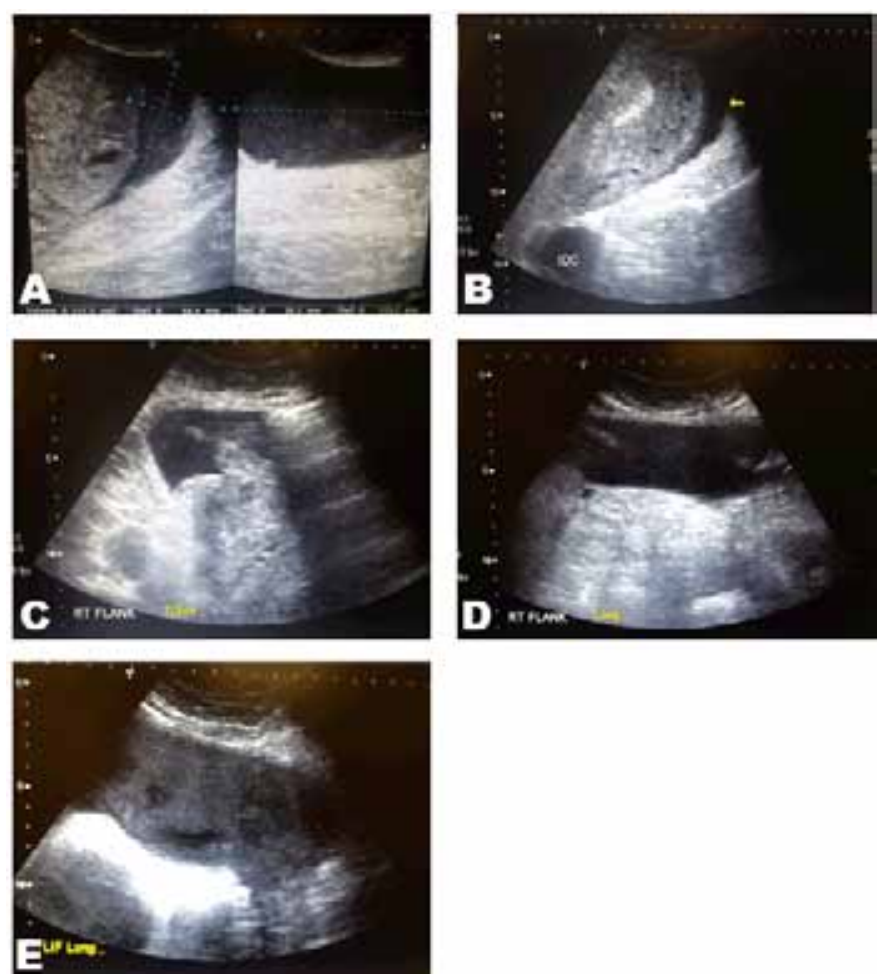

Figure 1: Ultrasound images. (A) Large pelvic collection behind the uterus, (B) Different view of collection behind uterus/ Pouch of Douglas, (C) Collection near the right kidney, (D) Longitudinal view of collection superior to the right kidney, and (E) Smaller collection on the left kidney.
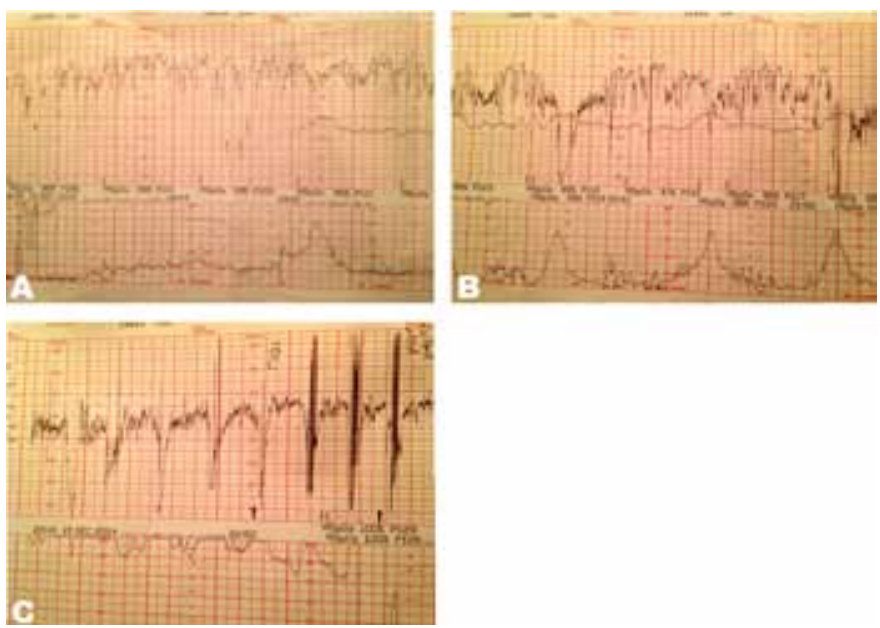

Figure 2: CTG images. (A) Trace before loss-of-consciousness and pre-ARM, (B) Trace after ARM with variable deceleration, (C) Trace with ongoing variable decelerations. 
Day 1 post-laparotomy, vitals of the patient were much improved. She was no longer tachycardic. Her pulse rate was 90 per minute and blood pressure was $120 / 60$ mmHg. Since the operation, her PR remained between 80-90 and she remained well. The only complication postoperatively, was an increase in haemoserous fluid in her abdominal drain 4 days after the operation which resolved on its own. She continued to improve postoperatively and was discharged home with her baby boy nine days after the operation.

\section{DISCUSSION}

Spontaneous hemoperitoneum in pregnancy is rare but if not diagnosed early can be life-threatening both to the mother and her baby. In 1950, a review of 75 cases due to utero-ovarian vessel rupture reported a $49 \%$ maternal mortality $[1,2]$. Though it can occur in all trimesters, $61 \%$ occurred before labor, $18 \%$ was intrapartum and $21 \%$ was postnatally [3]. Brosens et al. reviewed 25 cases of spontaneous hemoperitoneum in 12 publications and noted that $72 \%$ of them were in nulliparous patients with a mean age of 30 years $[4,5]$. These patients were noted to have endometriosis as a risk factor.

While there is no definite cause to these incidents, a few factors are thought to contribute to the increased likelihood of a spontaneous vessel rupture. These include dilated utero-ovarian veins which have tortuous nature, lack of valves, increasing physiological demands of pregnancy, muscular activities such as pushing phase of second stage labor that may cause sudden rise in venous pressure $[1,3]$. In the cases of patients with endometriosis, chronic inflammation due to endometriosis influenced by progesterone results in more fragile utero-ovarian vessels which could lead to its spontaneous rupture [4].

With the advancement of medicine, maternal mortality has been lowered to $3.6 \%[1,2]$. However, the diagnosis of this condition remains challenging because it is rarely made before an exploratory laparotomy and is most frequently misdiagnosed as placental abruption or a uterine rupture especially when it happens before labor without diagnostic imaging. Even then, the normally existing small number of ascites during pregnancy may be confusing in distinguishing early minimal internal bleeding [3]. Other differentials of this condition include rupture of the spleen or liver, perforated appendicitis, bleeding ectopic decidua, HELLP syndrome and splenic and hepatic artery aneurysm rupture. Percutaneous embolization of vessels is an option if diagnosed before laparotomy [9].

The most common sign and symptom of hemoperitoneum is abdominal pain, hypovolemic shock and a marked fall in the hemoglobin level. While ultrasonography for preoperative evaluation is important for obstetric cases with acute abdominal symptoms, its specificity as discussed above is low during pregnancy. Usually upon diagnosis, the amount of internal bleeding is extensive at emergency laparotomy and there is a need to perform cesarean section for fetal distress or on the presumption that there is a placental abruption. While pre-delivery diagnosis is important, contraction pain during labor can mask the peritoneal irritation from internal bleeding and delay diagnosis. It can even worsen the condition as the pushing force during second stage of labor may increase the risk of venous rupture $[1,3]$.

In our case, diagnosis of uterine surface vessel tear was made at laparotomy. Delay to diagnosis was due to several factors including a large BMI, the fact that she was a multigravida with prior normal deliveries and that she took time to become peritonitic. One identifiable risk that could have contributed to this condition was that she had undergone a previous oophorectomy in the same side where the uterine vessel tore. We hypothesize that the previous surgery may have caused adhesions in the area which then broke off particularly with changes in pressure during parturition and active second stage. This then led to the surface vessel tear that continued to bleed causing hemoperitoneum.

This patient had also presented with a prolonged labor for a multigravida. Though she had been contracting adequately her progress was poor. Early signs to look out for were the changes in CTG during labor. In the case report by $\mathrm{Wu}$ et al., they discussed about the spontaneous rupture of uterine vessel in twin pregnancy. The CTG was suspicious and the mother had acute abdomen. These led to their initial diagnosis of placental abruption, which then led to the final diagnosis upon emergency cesarean section for preterm twins. In our case, the changes in CTG though suspicious did not warrant an emergency cesarean section.

\section{CONCLUSION}

In conclusion, uterine surface vessel rupture is rare and difficult to diagnose. However with close observation to vital signs and symptoms, immediate resuscitative efforts could be taken once patient deterioration was noted which enabled prompt management with surgery. This led to a better outcome for the patient.

\section{Author Contributions}

Juliana Quay - Substantial contributions to conception and design, Acquisition of data, Analysis and interpretation of data, Drafting the article, Revising it critically for important intellectual content, Final approval of the version to be published

Azra Naseem - Analysis and interpretation of data, Revising it critically for important intellectual content, Final approval of the version to be published

George Kaladelfos - Analysis and interpretation of data, Revising it critically for important intellectual content, Final approval of the version to be published 


\section{Guarantor}

The corresponding author is the guarantor of submission.

\section{Conflict of Interest}

Authors declare no conflict of interest.

\section{Copyright}

(C) 2015 Juliana Quay et al. This article is distributed under the terms of Creative Commons Attribution License which permits unrestricted use, distribution and reproduction in any medium provided the original author(s) and original publisher are properly credited. Please see the copyright policy on the journal website for more information.

\section{REFERENCES}

1. Hodgkinson CP, Christensen RC. Hemorrhage from ruptured utero-ovarian veins during pregnancy; report of 3 cases and review of the literature. Am J Obstet Gynecol 1950 May;59(5):1112-7.

2. Ginsburg KA, Valdes C, Schnider G. Spontaneous utero-ovarian vessel rupture during pregnancy: three case reports and a review of the literature. Obstet Gynecol 1987 Mar;69(3 Pt 2):474-6.
3. Wu CY, Hwang JL, Lin $\mathrm{YH}$, Hsieh BC, Seow KM, Huang LW. Spontaneous hemoperitoneum in pregnancy from a ruptured superficial uterine vessel. Taiwan J Obstet Gynecol 2007 Mar;46(1):77-80.

4. Brosens IA, Fusi L, Brosens JJ. Endometriosis is a risk factor for spontaneous hemoperitoneum during pregnancy. Fertil Steril 2009 Oct;92(4):1243-5.

5. Doger E, Cakiroglu Y, Yildirim Kopuk S, Akar B, Caliskan E, Yucesoy G. Spontaneous rupture of uterine vein in twin pregnancy. Case Rep Obstet Gynecol 2013;2013:596707.

6. Janicki TI, David LJ, Skaf R. Massive and acute hemoperitoneum due to rupture of the uterine artery by erosion from an endometriotic lesion. Fertil Steril 2002 Oct;78(4):879-81.

7. Aziz U, Kulkarni A, Lazic D, Cullimore JE. Spontaneous rupture of the uterine vessels in pregnancy. Obstet Gynecol 2004 May;103(5 Pt 2):1089-91.

8. Wada S, Yoshiyuki F, Fujino T, Sato C. Uterine vein rupture at delivery as a delayed consequence of laparoscopic surgery for endometriosis: a case report. J Minim Invasive Gynecol 2009 Jul-Aug;16(4):510-2.

9. Díaz-Murillo R, Tobías-González P, López-Magallón S, Magdaleno-Dans F, Bartha JL. Spontaneous Hemoperitoneum due to Rupture of Uterine Varicose Veins during Labor Successfully Treated by Percutaneous Embolization. Case Rep Obstet Gynecol 2014;2014:580384.
Access full text article on other devices

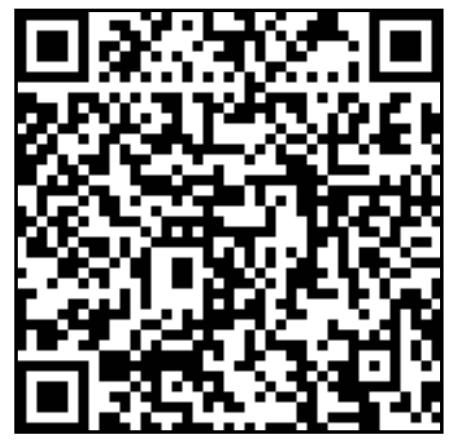

Access PDF of article on other devices

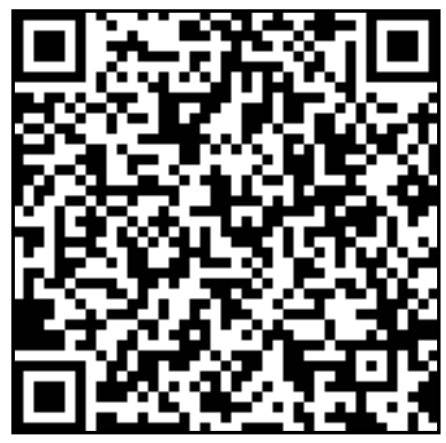

